\title{
Prioritizing otological surgery during the COVID-19 Pandemic
}

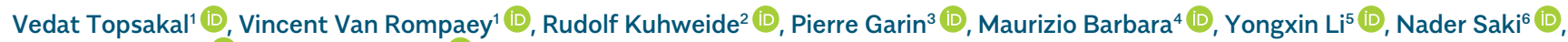 \\ Miguel Aristegui ${ }^{\text {(D) }}$, Thomas Somers ${ }^{8}$ (D) \\ 'Department of Otorhinolaryngology, Head and Neck Surgery, Antwerp University Hospital, Edegem, Antwerp, Belgium; University of Antwerp, \\ Faculty of Medicine and Health Sciences, Belgium \\ ${ }^{2}$ Department of ENT-HNS, AZ Sint-Jan Brugge-Oostende AV, Brugge, Belgium \\ ${ }^{3}$ ENT department, CHU UCL Namen, Mont-Godin, Belgium; Department of Anatomy, UNamur School of Medicine, Belgium \\ ${ }^{4}$ Sapienza University, Rome, Italy \\ ${ }^{5}$ Chinese Academy of Sciences, Beijing, Guangdong, China \\ ${ }^{6}$ Hearing Research Center, Ahvaz Jundishapur University of Medical Sciences, Ahvaz, Iran \\ ${ }^{7}$ Department of Otorhinolaryngology, Hospital General Universitario Gregorio Marañón, Madrid, Spain \\ ${ }^{8}$ Department of ENT-HNS, European Institute for Otorhinolaryngology- Head and Neck Surgery, Sint Augustinus, GZA Hospital, Antwerp, Belgium
}

Cite this article as: Topsakal V, Van Rompaey V, Kuhweide R, et al. Prioritizing otological surgery during the COVID-19 Pandemic. B-ENT 14 April 2020. 10.5152/B-ENT.2020.20126 [Epub Ahead of Print].

The initial cases of pulmonary infection with the novel corona virus SARS-CoV-2, causing COVID-19, occurred in Wuhan, Hubei Province, China in December 2019 and January 2020 (1). The spread through human-to-human transmission has led to a pandemic with disastrous consequences all over the world. The exponential rate of transmission and no existing vaccine has been a great challenge for all health care systems. A strategy to flatten the curve of transmission was put forward to adjust to the capacities of hospitals and particularly the intensive care units. Governments implemented isolation and social distancing upon societies either with laws or with strong recommendations.

As the pandemic proceeds anecdotal reports indicate that virus particles reside with extremely high concentrations in the nasal cavity and nasopharynx. Surgery involving respiratory epithelium is suddenly considered a significant risk factor for transmission (2). Evolving knowledge and experiences from China, Italy and Iran demonstrate that ENT (Ear, Nose and Throat) surgeons are amongst the highest risk professionals for contaminating themselves during their daily work.

Transmission is most probably via droplets from mucus or aerosolized particles when examining or operating in areas with contaminated epithelium and mucosae. In a sense middle ear mucosae and even the mastoid can be considered as part of the upper airway in terms of histological epithelium. Corona viruses have been detected in otitis media already in $1998(3,4)$.
However, little is known about the behaviour of the new SARSCoV-2 virus in the middle ear. Extra precautions seem necessary during otological surgery to prevent viral transmission, especially when high-speed drills are used together with irrigation. If a mastoid contains Sars-CoV-2 viral particles, classic mastoidectomy with a drill presents a great danger for aerosolizing particles in the operating theatre. At this moment of the COVID-19 pandemic there is a general belief that every health care worker in the operation theatre during mastoidectomy would be at risk.

Currently, many countries have issued local restrictions for the community with significant differences across the world. Correspondingly, many hospitals have local guidelines that are based on local resources and evolving insights. A guideline is by definition bound to a timeline and it seems during the COVID-19 pandemic these can change on a daily basis. As long as the supply of COVID-19 screening tests cannot meet the rising demand for testing or the interval between performing the swab and getting the result, the COVID-19 status of emergency cases may remain unknown. It seems obvious when there is no time or no capacity to test an emergency patient to consider these cases COVID-19 positive in both the outpatient clinic and the operation theatre.

This commentary aims to raise awareness amongst otolaryngologists in a difficult situation when confronted with patients that seem to require an "ontological" intervention. The

Corresponding Author: Vedat Topsakal; vedat.topsakal@uza.be

Received: April 8, 2020 Accepted: April 12, 2020 Available Online Date: April 14, 2020

Available online at www.b-ent.be 
authors do not claim to give guidelines and this manuscript is not intended for legal disputes. Most probably the listed possible scenarios are not even complete. However, clinical considerations and precautions are discussed for otological emergencies to help a clinician decide in difficult situations. The COVID-19 pandemic is not a time for medicine according to protocol but rather medicine according to reasoning.

Fortunately, acute life-threatening ear diseases requiring immediate surgery or even within 24 hours are infrequent. This is probably also the advantage of easy accessible health care systems in their normal functioning. Otological pathology is usually detected earlier and most often before it can lead to life threatening situations. During the COVID-19 pandemic there is a danger that patient with mild symptoms stay home and as the contamination risk continues over time, we could face more advanced otologic disease in emergency settings. Nevertheless, acute life-threatening complications of middle ear disease occur occasionally. Moreover, a classic mastoidectomy using a high-speed drill is a rather common procedure to gain access to the mastoid for several surgical treatments. In these cases, when there is no time to wait for COVID-19 screening test results, the patient should be considered potentially COVID-19 positive and the necessary procedure is performed under the best protective precautions given by the local hospital resources and guidelines. Even if the COVID-19 status is screened negative it might still be relevant to take precautions because of an alleged high false-negative rate. A larger study analysed a total of 1070 specimens of different types that were collected from 205 patients with COVID-19. (5) Bronchoalveolar lavage fluid specimens showed the highest positive rates (14 of 15; 93\%), followed by sputum (72 of 104; $72 \%$ ), nasal swabs (5 of $8 ; 63 \%$ ), fibro bronchoscope brush biopsy (6 of $13 ; 46 \%)$, pharyngeal swabs (126 of 398; 32\%), faeces (44 of $153 ; 29 \%$ ), and blood (3 of $307 ; 1 \%$ ). Usually, emergency imaging is performed of the temporal bone and a consideration could be to have a limited dose-screening CTscan of the thorax simultaneously. There have been reports that pulmonary COVID-19 lesions are easily recognized even in asymptomatic patients and are perhaps more sensitive than PCR screening tests (6). Although the surgeon is going to take COVID-19 precautions anyhow, this CT thorax could be of added value to the anaesthesiologist for the ventilation of the patient during the surgery. Experienced surgeons should best perform these procedures in absence of residents in training or interns to reduce surgical time and not to run the risk that an observing resident would be contaminated. In that sense no observers are allowed in theatre and the surgical team is best reduced to experienced staff in terms of anaesthesiologist, scrub nurse, circulating nurse and surgeon. All of them work under strict COVID-19 precautions as given by local hospital policies or guidelines of international professional societies or World Health Organization (WHO). The team should also pay attention to a strictly limited number of door movements and hazardous entries of unnecessary visitor/personnel in the theatre. Markings on the doors with "COVID-19 contaminated" are necessary precautions to warn personnel.

The surgeon should actively evaluate why and if the use of a high-speed drill is needed. The use of the high-speed drill is inevitably related with significant aerosolisation of bone and other tissues during drilling (7). Although transmission of viral particles is mainly through the respiratory system, there is some evidence of blood-borne transmission $(8,9)$. As experienced surgeons are used to treat HIV positive patients this risk is low, but present. Adequate hypotension facilitated by the anaesthesiologist will reduce bleeding tendency and reduce the related aerosolisation of blood and other mastoid fluids. An alternative could be the use of a piezoelectric drill, although the possible aerosolisation with such a device has never been quantified (10).

If possible, a chisel combined with curettes should be used for instance to facilitate drainage in acute mastoiditis instead of the drill. The infection often has weakened the bony cortex and once beyond tabula externa of the cortex the diploe is easily managed with curettes. FFP3 masks (with protective goggles or face-shield), or in case of limited resources FFP2, are advised by local protocols during surgery for the whole surgical team. The surgeon should consider wearing a face-shield because standard eye protection may not adequately prevent ocular transmission when the mastoidectomy is performed without microscope. Transmission through tears is perhaps not likely but there have been reports that speculate presence of Corona viruses in tears (11). When the use of the microscope is necessary the surgeon should not have a false sense of safety behind the microscope. The use of a face shield may be difficult behind the microscope, but perhaps customized self-made protection shields on the outer surface of the sterile microscope dress could be created. The use of exoscope, when available, should be considered. Even endoscopes may have an extra advantage nowadays for placing grommets or to perform paracentesis.

Table 1 summarizes a possible timeline for ear diseases requiring surgical intervention. Most of the treatments for these ear diseases have regional protocols and guidelines that might even differ between different otologic schools. As mentioned before, our aim was to generate awareness and advocate precautions. This table is best interpreted as a consideration at this stage of the COVID-19 pandemic and perhaps more specifically the stage the pandemic is in Belgium in April 2020. It is very likely that these timelines will shift within a month or two because COVID-19 will not leave after a month. After the peak of this pandemic, restrictions on daily otological practice should somehow be adjusted to allow health service closer to the standards we are used to (Table 1).

The relatively most frequent otological emergency that comes to mind is complicated acute otitis media. If mastoiditis is diagnosed by radiological imaging and the patient is stable and free from underlying condition that potentially compromises normal recovery, a medical therapy seems safe to be offered in the first 24 to 48 hours. Admission to the hospital with appropriate intravenous (IV) antibiotics is advisable and otorrhea is favoured to speed up evacuation. Introduction of a grommet is advised in many guidelines at this stage. However, when this would require general anaesthesia for instance in a paediatric patient there would be a risk for contamination. The risk of this contamination probably does not justify the possibly little benefit of the grommet to this patient. The main benefit will have to come from the IV antibiotics at this stage of the mastoiditis. A paracentesis under local anaesthesia could be considered as an alternative. 
Table 1. Priority of otologic surgery during Covid-19 pandemic

Priority: As Soon As Possible (ASAP)

- Life threatening compllications of ear disease

Priority: Within 48 to 72 hours

- Acute mastoiditis not responding to intravenous antibiotics

- Acute mastoiditis with subperiosteal abscess

- Cholesteatoma with facial nerve palsy

- Cholesteatoma with intracranial complication

- Barotrauma with evident perilymph fistula and SNHL

- Trauma to

Facial nerve

Pinna

- Hydrocephalus because of brainstem compression by vestibular schwannoma

Priority: Delay up to 4 weeks may seem safe for

- Cochlear implantation for SNHL because of meningitis

- Otologic Neoplasia; shared decision of multidisciplinary teams

Priority: Delay up to 12 weeks may seems safe for

- Cholesteatoma, uncomplicated and stable

- Cochlear implantation for pre-lingual profound SNHL

- Implantable hearing aids

- Non-life threatening lesions requiring neurotologic procedures

- Ossiculoplasty, Stapedoplasty, Meatoplasty, Tympanoplasty

- Grommets

March 2020 Belgium; SNHL: sensorineural hearing loss

When a mastoiditis is also complicated with a subperiostal abscess surgery seems necessary within 48-72 hours. If there are clinical signs of sepsis the surgery should be offered even faster, especially when a sepsis is present this surgery becomes an emergency. Basically, this example of acute mastoiditis demonstrates how table 1 should be interpreted: with reasoning and not blindly as a protocol. Many otological diseases can be safely deferred depending on the clinical presentation of the patient but much also depends on the comfort zone of the responsible surgeon. The treating surgeon is best suited to evaluate the treats of illness itself and the patient characteristics (including age, predisposing conditions, etc.) and weight this carefully to the risk of contamination the whole operation team. Main goals would be a safe working environment for health care workers and appropriate care for the patient.

Admission of a patient to hospital either for surgery or not, requires of course that hospital management provides clean areas for these patients and defines contaminated areas for COVID-19 positive patients and clean areas for COVID-19 negative patients. But still there are movements of patients requiring extra examinations and personnel are moving around as well. Elective interventions are therefore contra-indicated. This is not new in otology. When a patient is diagnosed with pneumonia all elective ear surgery is usually deferred. In case of a false negative COVID-19 test the mechanical ventilation during the surgery can worsen the COVID-19 status of a patient to a more severe stage. The COVID-19 infection is described as a 3-stage process: stage I, an asymptomatic incubation period with or without detectable virus; stage II, non-severe symptomatic period with the presence of virus; stage III, severe re- spiratory symptomatic stage with high viral load (12). Even the high risk of contamination of all otologic patient in a post-operative period by just being in the hospital makes it necessary to evaluate on a "day by day" basis and weigh the decision to indicate which procedure is really a vital emergency. Therefore table 1 should be interpreted in the specific scenario of each hospital with their specific precautions and capacities and in light of the local epidemiological stage as well.

Although table 1 is focussed on surgical interventions otologist might encounter some challenges in otological emergencies usually treated in an outpatient setting. For instance, there is a large consensus on the use of early oral steroid therapy for sudden sensorineural hearing loss ( $\mathrm{SNHL}$ ). This might require us to discuss this therapy with infectious disease specialist whether the COVID-19 status of the patient will allow this or not. The same will perhaps apply for idiopathic facial nerve palsy. Despite consensus on the use of early steroid therapy for idiopathic facial nerve palsy, the Covid-19 pandemic entails a general tendency not to start oral steroids until safety data is available.

Most outpatient activities are postponed for the moment for most ENT services except for head and neck oncology programs perhaps. However, sometimes there might be a risk for overlooking a potentially life-threatening disorder, e.g. when acute-onset vertigo is produced by a stroke in the posterior fossa and not by self-limiting vestibular neuritis. Hopefully the current restrictive measures will flatten the curve soon and otorhinolaryngologists can gradually resume daily practice.

Many disturbing reports have put otorhinolaryngologists amongst the clinicians most at risk of COVID-19 infection. At this moment we don't know why. Is it because ENT surgeons have worked with COVID-19 patients in a non-routine environment: screening at emergency and patient care on wards. Could it be that we contaminate ourselves because of non-routine tasks? Or, is it perhaps because of our previous routine tasks? More specifically an otorhinolaryngologist in a routine workflow in the outpatient clinic or even operating theatre will work with the upper airway. It seems likely that ENT surgeons have had exposure to several corona virus infections before in their career. Could it be that this makes us more prone to an extreme overreaction of the immune system by this new COVID-19 virus?

Probably these questions will be answered by ongoing or future studies. Meanwhile, table 1 is based upon the assumption that our routine work-related transmission risk is high. With progressing insight this table might even be obsolete tomorrow. For the time being, it gives some cohesion among otologists for helping our patient and protecting our team.

Peer-review: Externally peer-reviewed.

Author Contributions: Concept - V.T., V.V.R.; Design - V.T., V.V.R., R.K, P.G., M.B., Y.L., N.S., M.A., T.S.; Supervision V.T., V.V.R., R.K, P.G., M.B., Y.L., N.S., M.A., T.S.; Resources - V.T., V.V.R., R.K, P.G., M.B., Y.L., N.S., M.A., T.S..; Materials - X.X., X.X.; Data Collection and/or Processing V.T., V.V.R., R.K, P.G., M.B., Y.L., N.S., M.A., T.S.; Analysis and/or Interpretation - V.T., V.V.R., R.K, P.G., M.B., Y.L., N.S., M.A., T.S.; Literature Search - V.T., V.V.R., R.K, P.G., M.B., Y.L., N.S., M.A., T.S.; Writing Manuscript V.T.; Critical Review - V.T., V.V.R., R.K, P.G., M.B., Y.L., N.S., M.A., T.S. 
Conflict of Interest: The authors have no conflicts of interest to declare.

Financial Disclosure: The authors declared that this study has received no financial support.

\section{References}

1. Li Q, Guan X, Wu P, et al. Early transmission dynamics in Wuhan, China, of Novel Coronavirus-infected pneumonia. N Engl J Med. 2020: 382; 1199-207. [CrossRef]

2. Patel ZM, Fernandez-Miranda J, Hwang PH, et al. Precautions for endoscopic transnasal skull base surgery during the Covid-19 Pandemic. [accepted for publication in Neurosurgery, Oxford University Press. Epub 2020 Mar 23]

3. Pitkäranta A, Jero J, Arruda E, Virolainen A, Hayden FG. Polymerase chain reaction-based detection of rhinovirus, respiratory syncytial virus, and coronavirus in otitis media with effusion. J Pediatr. 1998: 133; 390-4. [CrossRef]

4. Pitkäranta A, Virolainen A, Jero J, Arruda E, Hayden FG. Detection of rhinovirus, respiratory syncytial virus, and coronavirus infections in acute otitis media by reverse transcriptase polymerase chain reaction. Pediatrics. 1998:102; 291-5. [CrossRef]

5. Wang W, Xu Y, Gao R, et al. Detection of SARS-CoV-2 in different types of clinical specimens. JAMA. 2020 Mar 11. [Epub Ahead of Print] [CrossRef]
6. Hu Z, Song C, Xu C, et al. Clinical Characteristics of 24 asymptomatic Infections with COVID-19 screened among close contacts in Nanjing, China. Sci China Life Sci. 2020 Mar 4. [Epub Ahead of Print] [CrossRef]

7. D L Jewett, P Heinsohn, C Bennett, A Rosen, C Neuilly. Blood-containing aerosols generated by surgical technique: a possible infectious hazard. Am Ind Hyg Assoc J. 1992: 53; 22831. [CrossRef]

8. Zhang W, Du RH, Li B, et al. Molecular and serological investigation of 2019-nCov infected patients. Emerg Microbes Infect. 2020: 9 ;386-389. [CrossRef]

9. Chang L, Yan Y, Wang L. Coronavirus Disease 2019: Coronaviruses and blood safety. Transfus Med Rev. 2020 Feb 21. [Epub ahead of print] [CrossRef]

10. Ito $T$, Mochizuki $H$, Watanabe $T$, et al. Safety of ultrasonic bone curette in ear surgery by measuring skull bone vibrations. Otol Neurotol. 2014: 35; e135-9. [CrossRef]

11. Xia J, Tong J, Liu M, Shen Y, Guo D. Evaluation of Coronavirus in tears and conjunctival secretions of patients with SARSCoV-2 Infection. J Med Virol. 2020 Feb 26. [Epub ahead of print] [CrossRef]

12. Shi $Y$, Wang $Y$, Shao $C$, et al. COVID-19 infection: the perspectives on immune responses. Shi Cell Death Differ. 2020 Mar 23. [Epub ahead of print] [CrossRef] 